\title{
Continuum-discretized coupled-channels calculations with core excitation
}

\author{
R. de Diego* \\ Departamento de Física Atómica, Molecular y Nuclear, Facultad de Física, Universidad de Sevilla, Apdo. 1065, E-41080 Sevilla, Spain and \\ Centro de Física Nuclear, Universidade de Lisboa, Av. Prof. Gama Pinto 2, P-1649-003 Lisboa, Portugal \\ J. M. Arias ${ }^{\dagger}$ J. A. Lay ${ }^{\ddagger}$ and A. M. Moro ${ }^{\S}$ \\ Departamento de Física Atómica, Molecular y Nuclear, Facultad de Física, Universidad de Sevilla, Apdo. 1065, E-41080 Sevilla, Spain
}

(Received 19 December 2013; revised manuscript received 5 May 2014; published 26 June 2014)

\begin{abstract}
The effect of core excitation in the elastic scattering and breakup of a two-body halo nucleus on a stable target nucleus is studied. The structure of the weakly bound projectile is described in the weak-coupling limit, assuming a particle-rotor model. The eigenfunctions and the associated eigenvalues are obtained by diagonalizing this Hamiltonian in a square-integrable basis (pseudostates). For the radial coordinate between the particle and the core, a transformed harmonic oscillator (THO) basis is used. For the reaction dynamics, an extension of the continuum-discretized coupled-channels (CDCC) method, which takes into account dynamic core excitation and de-excitation due to the presence of noncentral parts in the core-target interaction, is adapted to be used along with a pseudostates (PS) basis.
\end{abstract}

DOI: 10.1103/PhysRevC.89.064609

PACS number(s): 24.10.Eq, 25.60.Gc, 25.70.De, 27.20.+n

\section{INTRODUCTION}

Nuclei in the proximity of the proton and neutron driplines are often weakly bound, or even unbound, and hence their properties are influenced by positive-energy states. Collisions of these systems with stable nuclei will also be influenced by the coupling to the unbound states. This effect was first noticed in deuteron-induced reactions, and later observed in the scattering of other loosely bound nuclei, such as halo nuclei. Several formalisms have been developed to account for the effects of the coupling to breakup channels on reaction observables: continuum-discretized coupled-channels (CDCC) method [1,2], the adiabatic approximation [3,4], the AGS (Alt, Grassberger, and Sandhas) formulation of the Faddeev equations [5,6], and a variety of semiclassical approximations [7-12].

Typically, these approaches make use of a few-body description of the weakly bound nucleus. Furthermore, in their standard formulations, the constituent fragments are considered to be inert and, therefore, possible excitations of them are ignored. This is a good approximation for deuteron scattering, for which both constituents can be considered inert at the energies of interest in nuclear studies, but it is questionable for more complex systems. Moreover, bound and unbound states of the few-body system are considered to be well described by pure single-particle configurations. This approximation ignores possible admixtures of different core states in the wave functions of the complete projectile. These admixtures are known to be important, particularly in the case of welldeformed cores, as for example, in the ${ }^{11} \mathrm{Be}$ halo nucleus.

\footnotetext{
*raulez@cii.fc.ul.pt

†ariasc@us.es

†lay@us.es; Present address: Dipartimento di Fisica e Astronomia, Università di Padova, I-35131 Padova, Italy, and Istituto Nazionale di Fisica Nucleare, Sezione di Padova, I-35131 Padova, Italy.

§moro@us.es
}

In this work, we concentrate in two-body weakly bound nuclei composed of a core plus a valence particle. For such systems, core excitation effects in elastic breakup have been recently studied with an extension of the distorted wave Born approximation (DWBA) formalism, which includes them within a no-recoil approximation [13-15], referred to hereafter as no-recoil XDWBA. These calculations have shown that core excitation effects have a sizable influence on the magnitude of the breakup cross sections [13,14]. Moreover, these core excitation effects interfere with the valence excitation mechanism, altering the diffraction pattern in the resonant breakup angular distributions [15]. This method, being based in the Born approximation, ignores higher order effects (such as continuum-continuum couplings) and cannot be applied to describe the effect of breakup on elastic scattering.

The effect of core excitation on elastic scattering has also been studied [16], using an extension of the adiabatic model of Ref. [17]. The formalism was applied to ${ }^{8} \mathrm{~B}+{ }^{12} \mathrm{C}$, and some contributions due to ${ }^{7} \mathrm{Be}$ core excitations were found at large angles. Due to the use of the adiabatic approximation this method is, however, restricted to intermediate and high energies.

A recent attempt to incorporate core excitation effects within a full-fledged coupled-channels calculation was done in Ref. [18], using an extended version of the CDCC formalism (XCDCC). The method was applied to the scattering of oneneutron halo nuclei, using deformed valence-core and coretarget potentials to account for the core excitation mechanism. These calculations suggested a very small effect of core excitation, ${ }^{1}$ in contrast with the results of Refs. [13-15].

\footnotetext{
${ }^{1}$ Some equation errors and bugs in the programming of Ref. [18] (and subsequent related papers) were detected during the control checks of the present work. An erratum to Ref. [18] has already been published by its authors $[19,20]$. See also the comment in Sec. IV.A.
} 
In this work, we revisit the formulation of the XCDCC method of Ref. [18] and perform calculations for the elastic scattering and breakup of ${ }^{11} \mathrm{Be}$ on several targets at low and intermediate energies. The aim of this work is to provide an improved description of the reaction dynamics, as compared to the no-recoil DWBA method and also to pin down the effect of core excitation in elastic scattering and breakup. Our description of the reaction dynamics follows closely the derivation of Ref. [18], but a new code to compute the required coupling potentials has been developed in order to provide an independent assessment of the importance of core excitation effects in the scattering of halo nuclei. The main difference between our approach and that of Ref. [18] relies on the description of the states of the weakly bound projectile. In Ref. [18], the wave functions for these states were obtained by direct integration of the multichannel Schrödinger equation, subject to the appropriate boundary conditions for bound or unbound states. The latter were then grouped into bins, constructed by superposition of scattering states, following the standard average procedure. In this work, we use instead the so-called pseudostate method, in which the projectile states are approximated by the eigenstates of the Hamiltonian in a truncated basis of square-integrable functions. Negative-energy eigenvalues correspond to the bound states of the system, whereas those located at positive energies, usually referred to as pseudostates (PS), can be regarded as a finite and discrete representation of the continuum spectrum. The method has been successfully applied to two- [21-23] and three-body problems [24-27]. In particular, we make use of a transformed harmonic oscillator (THO) basis. This basis has been applied to the case of spherical systems [23] and also to deformed systems [28]. In both cases, the THO basis is used to describe the relative motion between the clusters and it is obtained by applying a local scale transformation (LST) to the harmonic oscillator (HO) basis. The LST, adopted from a previous work of Karataglidis et al. [29], is such that it transforms the Gaussian asymptotic behavior into an exponential form, thus ensuring the correct asymptotic behavior for the bound wave functions. The combined XCDCC + THO formalism is applied to ${ }^{11} \mathrm{Be}+p,{ }^{11} \mathrm{Be}+{ }^{64} \mathrm{Zn}$, and ${ }^{11} \mathrm{Be}+{ }^{208} \mathrm{~Pb}$ reactions and the effect of core excitation is discussed in each case (light, medium, and heavy target).

The work is structured as follows. In Sec. II we briefly recall the THO basis used for the description of two-body systems with core excitation. In Sec. III, the XCDCC formalism, particularized to our basis functions, is revisited. In Sec. IV the XCDCC + THO method is applied to several reactions induced by the ${ }^{11} \mathrm{Be}$ nucleus. Finally, in Sec. V the main results of this work are summarized.

\section{STRUCTURE OF THE PROJECTILE IN A THO BASIS}

In this section, we briefly review the features of the PS basis used in this work to describe the states of a two-body composite projectile, made of a valence particle $(v)$ and a core nucleus $(c)$ (see schematic in Fig. 1). The Hamiltonian of this system, $H_{p}$, is described in the weak-coupling limit and is

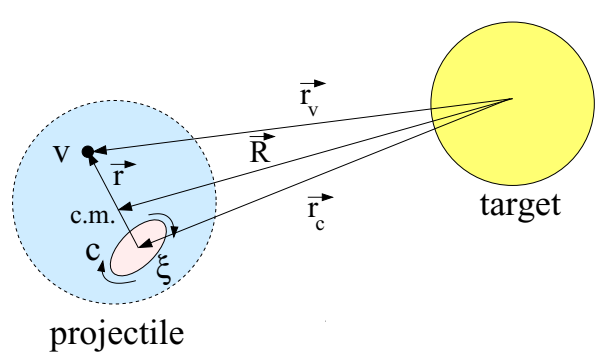

FIG. 1. (Color online) Schematic sketch of the weakly bound projectile composed by a core $(c)$ and a valence particle $(v)$. To study the scattering of the composite projectile with an inert target, within a three-body model, the relevant coordinates are the relative coordinate of the valence particle with respect to the core $(\vec{r})$ and that between the center of mass of the projectile and the target $(\vec{R})$. Note that the valence-target and core-target coordinates $\left(\vec{r}_{v}\right.$ and $\vec{r}_{c}$, respectively) can be written in terms of $\vec{r}$ and $\vec{R}$.

written as

$$
H_{p}(\vec{r}, \xi)=T(\vec{r})+V_{v c}(\vec{r}, \xi)+h_{c}(\xi),
$$

where $T(\vec{r})$ is the core-valence kinetic energy operator, $V_{v c}$ is the valence-core interaction, and $h_{c}(\xi)$ is the intrinsic Hamiltonian of the core.

In the calculations presented in this work, the composite system (projectile) is treated within the particle-rotor model [30]. Therefore, we assume that the core nucleus has a permanent deformation which, for simplicity, is taken to be axially symmetric. Thus, we can characterize the deformation by a single parameter, $\beta_{2}$. In the body-fixed frame, the surface radius is parameterized as $R(\hat{\xi})=R_{0}\left[1+\beta_{2} Y_{20}(\hat{\xi})\right]$, with $R_{0}$ as an average radius. Starting from a central potential, $V_{v c}^{(0)}(r)$, the full valence-core interaction is obtained by deforming this interaction as

$$
V_{v c}(\vec{r}, \hat{\xi})=V_{v c}^{(0)}\left(r-\delta_{2} Y_{20}(\hat{\xi})\right),
$$

with $\delta_{2}=\beta_{2} R_{0}$, usually called deformation length. By transforming this to the space-fixed reference frame and expanding the spherical harmonics, this deformed potential reads (see, e.g., Ref. [31])

$$
V_{v c}(r, \theta, \phi)=\sqrt{4 \pi} \sum_{\lambda \mu} \mathcal{V}_{v c}^{\lambda}(r) \mathcal{D}_{\mu 0}^{\lambda}(\alpha, \beta, \gamma) Y_{\lambda \mu}(\hat{r})
$$

with the radial form factors

$$
\mathcal{V}_{v c}^{\lambda}(r)=\frac{\hat{\lambda}}{2} \int_{-1}^{1} V_{v c}\left(r-\delta_{2} Y_{20}\left(\theta^{\prime}, 0\right)\right) P_{\lambda}(u) d u,
$$

(with $u=\cos \theta^{\prime}$ and $\hat{\lambda} \equiv \sqrt{2 \lambda+1}$ ). $\mathcal{D}_{\mu 0}^{\lambda}(\alpha, \beta, \gamma)$ is a rotation matrix, depending on the Euler angles $\{\alpha, \beta, \gamma\}$ which define the transformation from the body-fixed frame to the laboratory frame.

The eigenstates of the Hamiltonian and their associated wave functions can be obtained by solving a system of differential equations, as done in Ref. [18]. Alternatively, they can be obtained by diagonalizing the Hamiltonian matrix in a finite basis of square integrable functions. In this work we use 
this second procedure. For that, we choose a basis of the form

$$
\phi_{i, \alpha, J_{p}, M_{p}}^{T H O}(\vec{r}, \xi)=R_{i, \ell}^{T H O}(r)\left[\mathcal{Y}_{(\ell s) j}(\hat{r}) \otimes \varphi_{I}(\xi)\right]_{J_{p} M_{p}},
$$

where the label $\alpha$ denotes the set of quantum numbers $\{\ell, s, j, I\}$, with $\vec{\ell}$ (valence-core orbital angular momentum) and $\vec{s}$ (spin of the valence) both coupled to $\vec{j}$ (total valence particle angular momentum). The total spin of the projectile, $\vec{J}_{p}$, is given by the coupling between $\vec{j}$ and $\vec{I}$ (intrinsic spin of the core). The valence-core relative motion is described by the functions $R_{i, \ell}^{T H O}(r)$ (radial part) and $\mathcal{Y}_{(\ell s) j}(\hat{r})$ (spin-angular part), while the functions $\varphi_{I}(\xi)$ describe the core states. The functions $R_{i, \ell}^{T H O}(r)$ are generated by applying a local scale transformation (LST) to the spherical HO basis functions,

$$
R_{i, \ell}^{T H O}(r)=\sqrt{\frac{d s}{d r}} R_{i, \ell}^{H O}[s(r)],
$$

where $R_{i, \ell}^{H O}[s(r)]$ (with $i=1,2, \ldots$ ) is the radial part of the HO functions and $s(r)$ defines the LST. For the latter we use the analytical prescription by Karataglidis et al. [29]

$$
s(r)=\frac{1}{\sqrt{2} b}\left[\left(\frac{1}{r}\right)^{m}+\left(\frac{1}{\gamma \sqrt{r}}\right)^{m}\right]^{-\frac{1}{m}},
$$

that depends on the parameters $m, \gamma$ and the oscillator length $b$. This transformation was shown in Ref. [29] to depend weakly on $m$. The value $m=4$ was proposed in Ref. [29] and adopted here. Thus, the adopted LST depends on $\gamma$ and $b$. The ratio $\gamma / b$ determines the range of the basis functions and the density of eigenstates as a function of the excitation energy. As $\gamma / b$ decreases, the basis functions explore larger distances and the corresponding eigenvalues concentrate at smaller excitation energies.

The eigenstates of the Hamiltonian (1) are expressed as an expansion in the THO basis,

$$
\Phi_{n, J_{p}, M_{p}}^{(N)}(\vec{r}, \xi)=\sum_{i=1}^{N} \sum_{\alpha} C_{i, \alpha, J_{p}}^{n} \phi_{i, \alpha, J_{p}, M_{p}}^{T H O}(\vec{r}, \xi),
$$

where $N$ is the number of radial functions retained in the truncated THO basis, $n$ is an index identifying each eigenstate, and $C_{i, \alpha, J_{p}}^{n}$ are the expansion coefficients of the pseudostates in the truncated basis. The sum in $i$ can be performed to get

$$
\Phi_{n, J_{p}, M_{p}}^{(N)}(\vec{r}, \xi)=\sum_{\alpha} \frac{u_{n, \alpha}^{J_{p}}(r)}{r}\left[\mathcal{Y}_{(\ell s) j}(\hat{r}) \otimes \varphi_{I}(\xi)\right]_{J_{p} M_{p}}
$$

with

$$
u_{n, \alpha}^{J_{p}}(r)=r \sum_{i=1}^{N} C_{i, \alpha, J_{p}}^{n} R_{i, \ell}^{T H O}(r) .
$$

The negative eigenvalues of the Hamiltonian (1) are identified with the energies of bound states whereas the positive ones correspond to a discrete representation of the continuum spectrum.

\section{SCATTERING FRAMEWORK}

Once the projectile wave functions have been obtained, we proceed to solve the three-body scattering problem. The formalism has been derived and presented in detail in Ref. [18] so we summarize here the main formulas and adapt them to our PS scheme. We express the three-body wave functions $\Psi_{J_{T}, M_{T}}$ in terms of the set $\left\{\Phi_{n, J_{p}}^{(N)}\right\}$ :

$$
\Psi_{J_{T}, M_{T}}(\vec{R}, \vec{r}, \xi)=\sum_{\beta} \chi_{\beta}^{J_{T}}(R)\left[Y_{L}(\hat{R}) \otimes \Phi_{n, J_{p}}^{(N)}(\vec{r}, \xi)\right]_{J_{T}, M_{T}},
$$

where, in addition to the projectile coordinates $\vec{r}$ and $\xi$, we have the relative coordinate $\vec{R}$ between the projectile center of mass and the target (assumed to be structureless); see Fig. 1. The different quantum numbers are labeled by $\beta=\left\{L, J_{p}, n\right\}$, where $\vec{L}$ (projectile-target orbital angular momentum) and $\vec{J}_{p}$ both couple to the total spin of the three-body system $\vec{J}_{T}$. The spin of the target is ignored for simplicity of the notation.

The radial coefficients, $\chi_{\beta}^{J_{T}}(R)$, from which the scattering observables are extracted, are calculated by inserting Eq. (11) in the Schrödinger equation, giving rise to a system of coupled differential equations. The main physical ingredients of these coupled equations are the coupling potentials:

$$
U_{\beta, \beta^{\prime}}^{J_{T}}(R)=\left\langle\beta ; J_{T}\left|V_{c t}(\vec{R}, \vec{r}, \xi)+V_{v t}(\vec{R}, \vec{r})\right| \beta^{\prime} ; J_{T}\right\rangle,
$$

where we follow the notation used in Ref. [18],

$$
\left\langle\hat{R}, \vec{r}, \xi \mid \beta ; J_{T}\right\rangle=\left[Y_{L}(\hat{R}) \otimes \Phi_{n, J_{p}}^{(N)}(\vec{r}, \xi)\right]_{J_{T}}
$$

The valence particle-target interaction $\left(V_{v t}\right)$ is assumed to be central and will be represented by a phenomenological optical potential describing the valence particle-target elastic scattering at the appropriate energy per nucleon. On the other hand, the core-target interaction is assumed to contain a noncentral part, responsible for the dynamic core excitation and de-excitation mechanism. In general, this interaction can be expressed in the multipolar form:

$$
V_{c t}(\vec{R}, \vec{r}, \xi)=V_{c t}\left(\vec{r}_{c}, \xi\right)=\sqrt{4 \pi} \sum_{Q q} V_{Q q}\left(r_{c}, \xi\right) Y_{Q q}\left(\hat{r}_{c}\right),
$$

where $\vec{r}_{c}=\vec{R}-a \vec{r}$ (see Fig. 1), with $a=m_{v} /\left(m_{v}+m_{c}\right.$ ) $\left(m_{c}\right.$ and $m_{v}$ denote the core and valence particle masses, respectively).

In some models, such as in the rotational model assumed here, the multipole terms $V_{Q q}\left(r_{c}, \xi\right)$ factorize into a radial part and a structure part, i.e.,

$$
V_{c t}(\vec{R}, \vec{r}, \xi)=\sqrt{4 \pi} \sum_{Q q} \mathcal{V}_{c t}^{Q}\left(r_{c}\right) \mathcal{T}_{Q q}^{*}(\xi) Y_{Q q}\left(\hat{r}_{c}\right)
$$

Note that $V_{c t}(\vec{R}, \vec{r}, \xi)$ will contain, in general, both Coulomb and nuclear parts, so in this formalism both interactions are treated simultaneously. The matrix elements (12) were explicitly evaluated in Ref. [18], giving rise to the expression

$$
\begin{aligned}
U_{\beta: \beta^{\prime}}^{J_{T}}(R)= & \hat{L} \hat{L}^{\prime} \hat{J}_{p} \hat{J}_{p}^{\prime}(-1)^{J_{p}+J_{T}} \sum_{\Lambda}(-1)^{\Lambda} \hat{\Lambda}^{2} \\
& \times\left(\begin{array}{ccc}
\Lambda & L & L^{\prime} \\
0 & 0 & 0
\end{array}\right)\left\{\begin{array}{ccc}
J_{p} & J_{p}^{\prime} & \Lambda \\
L^{\prime} & L & J_{T}
\end{array}\right\} F_{J_{p} n: J_{p}^{\prime} n^{\prime}}^{\Lambda}(R) .
\end{aligned}
$$


The form factors $F_{J_{p} n: J_{p}^{\prime} n^{\prime}}^{\Lambda}(R)$ are given by

$$
F_{J_{p} n: J_{p}^{\prime} n^{\prime}}^{\Lambda}(R)=\sum_{K Q \lambda \alpha, \alpha^{\prime}} \mathcal{R}_{\alpha n: \alpha^{\prime} n^{\prime}}^{K Q \lambda}(R) P_{\alpha: \alpha^{\prime}}^{K Q \lambda: \Lambda},
$$

with the radial integrals:

$$
\mathcal{R}_{\alpha n: \alpha^{\prime} n^{\prime}}^{K Q \lambda}(R)=\hat{K} \int u_{n, \alpha}^{J_{p} *}(r) \mathcal{V}_{c t}^{Q K}(r, R) R^{\lambda}(a r)^{Q-\lambda} u_{n^{\prime}, \alpha^{\prime}}^{J^{\prime}}(r) d r
$$

where

$$
\mathcal{V}_{c t}^{Q K}(r, R)=\frac{1}{2} \int_{-1}^{+1} \frac{\mathcal{V}_{c t}^{Q}\left(r_{c}\right)}{r_{c}^{Q}} P_{K}(u) d u ; u=\hat{R} \cdot \hat{r} .
$$

The coefficients $P_{\alpha: \alpha^{\prime}}^{K Q \lambda: \Lambda}$ are explicitly written as

$$
\begin{aligned}
P_{\alpha: \alpha^{\prime}}^{K Q \lambda: \Lambda}= & (-1)^{j^{\prime}+\ell+\ell^{\prime}+s+Q} \hat{Q}^{2} \hat{K} \hat{j} \hat{j}^{\prime} \hat{\ell} \hat{\ell}^{\prime}\left(\begin{array}{ccc}
K & \lambda & \Lambda \\
0 & 0 & 0
\end{array}\right) \sqrt{\frac{(2 Q) !}{(2 \lambda) ![2(Q-\lambda)] !}}\left\langle I\left\|\mathcal{T}_{Q}(\xi)\right\| I^{\prime}\right\rangle \\
& \times \sum_{\Lambda^{\prime}} \hat{\Lambda}^{\prime 2}\left(\begin{array}{ccc}
K & Q-\lambda & \Lambda^{\prime} \\
0 & 0 & 0
\end{array}\right)\left(\begin{array}{ccc}
\Lambda^{\prime} & \ell & \ell^{\prime} \\
0 & 0 & 0
\end{array}\right)\left\{\begin{array}{ccc}
\Lambda^{\prime} & \Lambda & Q \\
\lambda & Q-\lambda & K
\end{array}\right\}\left\{\begin{array}{ccc}
j & j^{\prime} & \Lambda^{\prime} \\
\ell^{\prime} & \ell & s
\end{array}\right\}\left\{\begin{array}{ccc}
J_{p} & J_{p}^{\prime} & \Lambda \\
j & j^{\prime} & \Lambda^{\prime} \\
I & I^{\prime} & Q
\end{array}\right\},
\end{aligned}
$$

which, in addition to geometric coefficients, contain the structure reduced matrix elements $\left\langle I\left\|\mathcal{T}_{Q}(\xi)\right\| I^{\prime}\right\rangle$. Specific models enter into these expressions through the radial form factors $\mathcal{V}_{c t}^{Q}\left(r_{c}\right)$ and the structure reduced matrix elements. We give explicit expressions of these magnitudes for the model used in the calculations presented in this work.

For the Coulomb part of the core-target interaction, we use the usual multipole expansion

$$
V_{c t}^{\text {coul }}\left(\vec{r}_{c}, \xi\right)=\sum_{Q, q} \frac{4 \pi}{2 Q+1} \frac{Z_{t} e}{r_{c}^{Q+1}} \mathcal{M}(E Q q) Y_{Q q}\left(\hat{r}_{c}\right),
$$

where $\mathcal{M}(E Q q)$ is the multipole electric operator. Compared with the general expression (14) we have

$$
V_{Q q}\left(r_{c}, \xi\right) \equiv \frac{\sqrt{4 \pi}}{2 Q+1} \frac{Z_{t} e}{r_{c}^{Q+1}} \mathcal{M}(E Q q) .
$$

For the nuclear part of the core-target interaction, we follow the same approach as for the valence-core interaction, that is, we start with a central interaction, $V_{c t}^{(0)}\left(r_{c}\right)$, that is deformed assuming a quadrupole deformation characterized by a deformation length $\delta_{2}$. The resulting potential is expanded in spherical harmonics and transformed to the laboratory frame, giving rise to the result analogous to that of Eq. (3),

$$
V_{c t}^{\mathrm{nuc}}\left(r_{c}, \theta, \phi\right)=\sqrt{4 \pi} \sum_{Q q} \mathcal{V}_{c t}^{Q}\left(r_{c}\right) \mathcal{D}_{q 0}^{Q}\left(\alpha^{\prime}, \beta^{\prime}, \gamma^{\prime}\right) Y_{Q q}\left(\hat{r}_{c}\right),
$$

with $\left\{\alpha^{\prime}, \beta^{\prime}, \gamma^{\prime}\right\}$ the corresponding Euler angles and the radial form factors

$$
\mathcal{V}_{c t}^{Q}\left(r_{c}\right)=\frac{\hat{Q}}{2} \int_{-1}^{1} V_{c t}^{\mathrm{nuc}}\left(r_{c}-\delta_{2} Y_{20}\left(\theta^{\prime}, 0\right)\right) P_{Q}(u) d u,
$$

with $u=\cos \left(\theta^{\prime}\right)$. In comparison with Eq. (15) we have $\mathcal{T}_{Q q} \equiv$ $\mathcal{D}_{q 0}^{Q *}$. The reduced matrix elements of this operator are to be calculated between rotational states belonging to a rotational band characterized by a projection of $I$ along the symmetry axis $K$. Explicitly (see, e.g., [32]),

$$
\left\langle K I\left\|\mathcal{D}^{Q *}\right\| K I^{\prime}\right\rangle=\hat{I}^{\prime}\left\langle I^{\prime} K Q 0 \mid I K\right\rangle,
$$

where the convention of Bohr and Mottelson [30] for reduced matrix elements has been assumed.

\section{APPLICATION TO ${ }^{11}$ Be REACTIONS}

As an illustration of the formalism presented in the preceding sections, we consider the scattering of the halo nucleus ${ }^{11} \mathrm{Be}$ on ${ }^{1} \mathrm{H},{ }^{64} \mathrm{Zn}$, and ${ }^{208} \mathrm{~Pb}$ targets, comparing with available data for these reactions. The bound and unbound states of the ${ }^{11} \mathrm{Be}$ are known to contain significant admixtures of core-excited components [33-35], and hence core excitation effects are expected to be important. This has been in fact confirmed in the case of resonant breakup of this nucleus on ${ }^{1} \mathrm{H}[13,14]$ and ${ }^{12} \mathrm{C}$ targets [15], using the no-recoil XDWBA method.

As in previous works [13], the ${ }^{11} \mathrm{Be}$ structure is described with the particle-rotor model of Bohr and Mottelson with the Hamiltonian of Ref. [36] (model Be12-b), which consists of a Woods-Saxon central part, with a fixed geometry $(R=$ $2.483 \mathrm{fm}, a=0.65 \mathrm{fm})$ and a parity-dependent strength $\left(V_{c}=\right.$ $-54.24 \mathrm{MeV}$ for positive-parity states and $V_{c}=-49.67 \mathrm{MeV}$ for negative-parity ones). The potential contains also a spinorbit term, whose radial dependence is given by the derivative of the central Woods-Saxon part, and strength $V_{s o}=8.5 \mathrm{MeV}$. For the ${ }^{10} \mathrm{Be}$ core, this model assumes a permanent quadrupole deformation $\beta_{2}=0.67$ (i.e., $\delta_{2}=\beta_{2} R=1.664 \mathrm{fm}$ ). Only the ground state $\left(0^{+}\right)$and the first excited state $\left(2^{+}, E_{x}=\right.$ $3.368 \mathrm{MeV}$ ) are included in the model space. For the valencecore orbital angular momentum, we consider the values $\ell \leqslant 3$.

The particle-rotor model that we assumed may seem unrealistic, but it has been proved to provide a reasonable description of ${ }^{11} \mathrm{Be}[13,37,38]$ and other nuclei, such as several 
odd carbon isotopes [18,37,39]. More realistic descriptions of

${ }^{11} \mathrm{Be}$ have indeed been proposed in the literature (see, e.g., Refs. [40-42]) but the use of these more sophisticated structure models would make the evaluation of the coupling potentials much more involved (this can be seen for example in the microscopic cluster model recently applied to the description of the scattering of ${ }^{7} \mathrm{Li}$ [43]). For the purpose of the present work, we believe that the assumed rotor model provides a simple but still reliable choice.

To generate the THO basis for all the studied cases here, we use the LST of Eq. (7) with $m=4$ and $b=1.6 \mathrm{fm}$. The number of oscillator functions $N$ and the $\gamma$ parameter are determined specifically for each reaction and are specified below.

\section{A. Application to ${ }^{11} \mathrm{Be}+p$ resonant breakup}

We first apply the XCDCC + THO method to the breakup of ${ }^{11} \mathrm{Be}$ on a proton target at $63.7 \mathrm{MeV} /$ nucleon and compare with the data of Ref. [44]. The measured data consist in angular distributions for two intervals of the neutron- ${ }^{10} \mathrm{Be}$ core energy: (i) $E_{\text {rel }}=0-2.5 \mathrm{MeV}$ and (ii) $E_{\text {rel }}=2.5-$ $5.0 \mathrm{MeV}$. The first interval contains a narrow $5 / 2^{+}$resonance at $E_{\text {rel }}=1.28 \mathrm{MeV}$ [45]. This resonance has a dominant ${ }^{10} \mathrm{Be}\left(0^{+}\right) \otimes v 1 d_{5 / 2}$ parentage and a small ${ }^{10} \mathrm{Be}\left(2^{+}\right) \otimes v 2 s_{1 / 2}$ component. The cross section for the second interval contains presumably contributions coming from several resonances, namely, $E_{x}=2.64 \mathrm{MeV}\left(3 / 2^{-}\right), 3.40 \mathrm{MeV}\left(3 / 2^{-}, 3 / 2^{+}\right)$, $3.89 \mathrm{MeV}\left(5 / 2^{-}\right)$, and $3.95 \mathrm{MeV}\left(3 / 2^{-}\right)$[45]. Previous calculations $[13,14]$, based on the no-recoil XDWBA method, showed indeed that the main contribution to the lower energy angular distribution arises from the single-particle excitation mechanism populating the $5 / 2_{1}^{+}$resonance, whereas for the higher energy angular distribution the main contribution comes from the excitation of the $3 / 2_{1}^{+}$resonance due to the collective excitation of the ${ }^{10} \mathrm{Be}$ core.

We repeat the calculations of Refs. [13,14] using the more sophisticated XCDCC formalism for the reaction dynamics and the THO PS basis for the ${ }^{11} \mathrm{Be}$ states. The LST was generated with the parameter $\gamma=1.6 \mathrm{fm}^{1 / 2}$. The number of oscillator functions was $N=14$. Continuum states with $J_{p}=1 / 2^{ \pm}, 3 / 2^{ \pm}$, and $5 / 2^{+}$were found to be enough for convergence of the calculated observables. The proton-neutron interaction was represented by a simple Gaussian interaction derived in Ref. [14], while for the core-target potential we used the CH89 optical model parametrization [46], but modified the real and imaginary depths in order to reproduce the experimental available elastic and inelastic data of ${ }^{10} \mathrm{Be}+p$ at 59.2 MeV/nucleon [47]. In order to reproduce the magnitude of the inelastic data, this potential required a deformation length of $\delta_{2}=1.9 \mathrm{fm}$, which is somewhat larger than the deformation used in our adopted rotor model for ${ }^{11} \mathrm{Be}$ but consistent with the values extracted in the DWBA analysis done in Ref. [47] for the same data. For the nuclear part, both monopole $Q=0$ and quadrupole $Q=2$ terms were included. For the Coulomb part, following the expression given in Eq. (21), both terms are also considered even though the $Q=0$ term gives the main contribution for such a light system. The value of $\left\langle 0^{+}\|\mathcal{M}(E 2)\| 2^{+}\right\rangle$was derived from the experimental value of $B\left(E 2 ; 0^{+} \rightarrow 2^{+}\right)=53(6) e^{2} \mathrm{fm}^{4}$

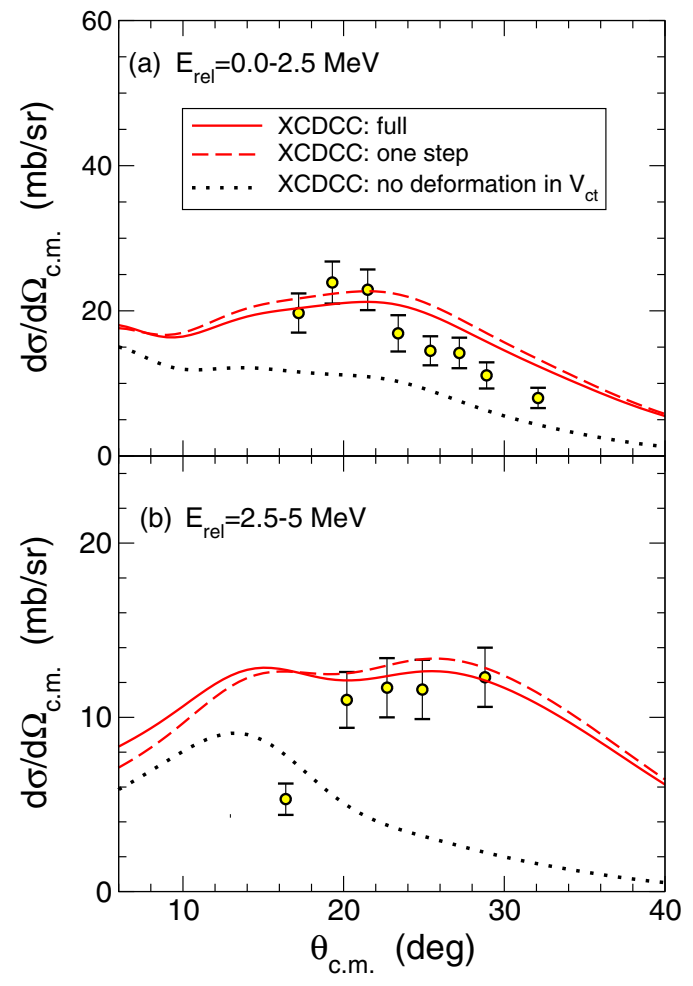

FIG. 2. (Color online) Differential breakup cross sections, with respect to the outgoing ${ }^{11} \mathrm{Be}^{*} \mathrm{c} . \mathrm{m}$. scattering angle, for the breakup of ${ }^{11} \mathrm{Be}$ on protons at $63.7 \mathrm{MeV} /$ nucleon. Top and bottom panels correspond to the neutron-core relative energy intervals $E_{\mathrm{rel}}=0$ $2.5 \mathrm{MeV}$ and $E_{\text {rel }}=2.5-5 \mathrm{MeV}$, respectively.

[48]. The reorientation term $2^{+} \leftrightarrow 2^{+}$was also included, and the value of the reduced matrix element $\left\langle 2^{+}\|\mathcal{M}(E 2)\| 2^{+}\right\rangle$ was derived from the computed value of $\left\langle 0^{+}\|\mathcal{M}(E 2)\| 2^{+}\right\rangle$, assuming that the $0^{+}$and $2^{+}$states of ${ }^{10} \mathrm{Be}$ are members of the same rotational band with $K^{\pi}=0^{+}$.

The results of these calculations are shown in Fig. 2. The solid line corresponds to our full coupled-channels calculation, including couplings to all orders. Considering the experimental error bars, the agreement with the data is fairly reasonable for both energy intervals, except for the first data point in the higher energy interval. These results are qualitatively similar to those found in Refs. $[13,14]$ using the no-recoil XDWBA approach.

To illustrate the importance of higher-order effects, we include also the first-order calculation assuming a one-step breakup mechanism (dashed lines). Differences between the latter and the full calculations are small but not negligible, indicating that, even at these relatively high incident energies, higher order effects are significant and hence an accurate description of these reactions require going beyond the simpler DWBA approximation. We also include the results obtained omitting the $Q=2$ term in the nuclear part of the core-target interaction, but keeping the deformation in the neutron-core interaction (dotted line). This result differs significantly from the full calculation, indicating very clearly the sizable effect of the dynamic core excitation mechanism during the collision. The difference is particularly noticeable in the higher energy 
interval, due to the fact that this interval is dominated by the $3 / 2^{+}$resonance, which is mostly populated by a core excitation mechanism [13].

It is worth noting that our results differ qualitatively from those performed in Ref. [49] for the same reaction, using also the XCDCC formalism, but with a binning discretization scheme. In benchmarking the results of the present work with those from Ref. [49], several mistakes were found in the equations of that reference, as well as in their numerical implementation. These mistakes result in a significant underestimation of core excitation effects $[19,20]$. In addition, some differences are expected due to the different choice of the $p-n$ interaction.

We have studied also the dependence of the core excitation effect with the incident energy. For this, we have performed additional XCDCC calculations for the same reaction at 10 $\mathrm{MeV} /$ nucleon and $200 \mathrm{MeV} /$ nucleon. At both energies, we use the $\mathrm{CH} 89$ parametrization for the ${ }^{10} \mathrm{Be}+p$ interaction. Because of the lack of experimental data at those energies, we keep the deformation length obtained from the fit of the inelastic data performed at $59.2 \mathrm{MeV}\left(\delta_{2}=1.9 \mathrm{fm}\right)$, and adjust the potential depths in order to reproduce the elastic and inelastic scattering of ${ }^{10} \mathrm{Be}+p$ obtained with a microscopic folding potential, generated with the effective nucleon-nucleon interaction of Jeukenne, Lejeune, and Mahaux (JLM) [50] and transition densities from antisymmetrized molecular dynamics (AMD) calculations (see Ref. [51] for a similar approach). The results are shown in Fig. 3. The calculations shown in the middle panels are just the same as those shown in Fig. 2, but are included here to facilitate the comparison using a wider angular range. One can see that the core excitation energy is important at the three incident energies, particularly in the region containing the $3 / 2^{+}$resonance.

\section{B. Application to ${ }^{11} \mathrm{Be}+{ }^{64} \mathrm{Zn}$ elastic and breakup}

As a second example, we consider the ${ }^{11} \mathrm{Be}+{ }^{64} \mathrm{Zn}$ reaction at 28.7 MeV. Quasielastic (elastic + inelastic) and inclusive breakup data from this reaction have been reported in Ref. [52] and have been analyzed within the standard CDCC framework in several works [53-55].

We compare these data with XCDCC calculations. For the neutron-target interaction, we used the same optical potential used in Ref. [54]. For the ${ }^{10} \mathrm{Be}+{ }^{64} \mathrm{Zn}$ interaction, we started from the optical potential derived in Ref. [52] from a fit of the elastic scattering data for this system. To account for the core excitation mechanism, this potential is deformed with the same deformation length used in the structure model, i.e., $\delta_{2}=1.664$ $\mathrm{fm}$. Coulomb multipoles (monopole and quadrupole) were also included, as in the previous case, according to the expansion (21). To recover the description of the ${ }^{10} \mathrm{Be}+{ }^{64} \mathrm{Zn}$ elastic data, once these additional couplings are included, the optical potential depths were readjusted, giving rise to the modified values $V_{0}=-84.5 \mathrm{MeV}$ and $W_{v}=-34.1 \mathrm{MeV}$, for the real and imaginary parts, respectively.

For the ${ }^{11} \mathrm{Be}$ projectile, continuum states up to $J_{p}=7 / 2$ (both parities) were included. These states were obtained by diagonalizing the ${ }^{11} \mathrm{Be}$ Hamiltonian in a THO basis with $N=10$ radial functions, $\ell \leqslant 3$ and $I=0,2$. For the LST,
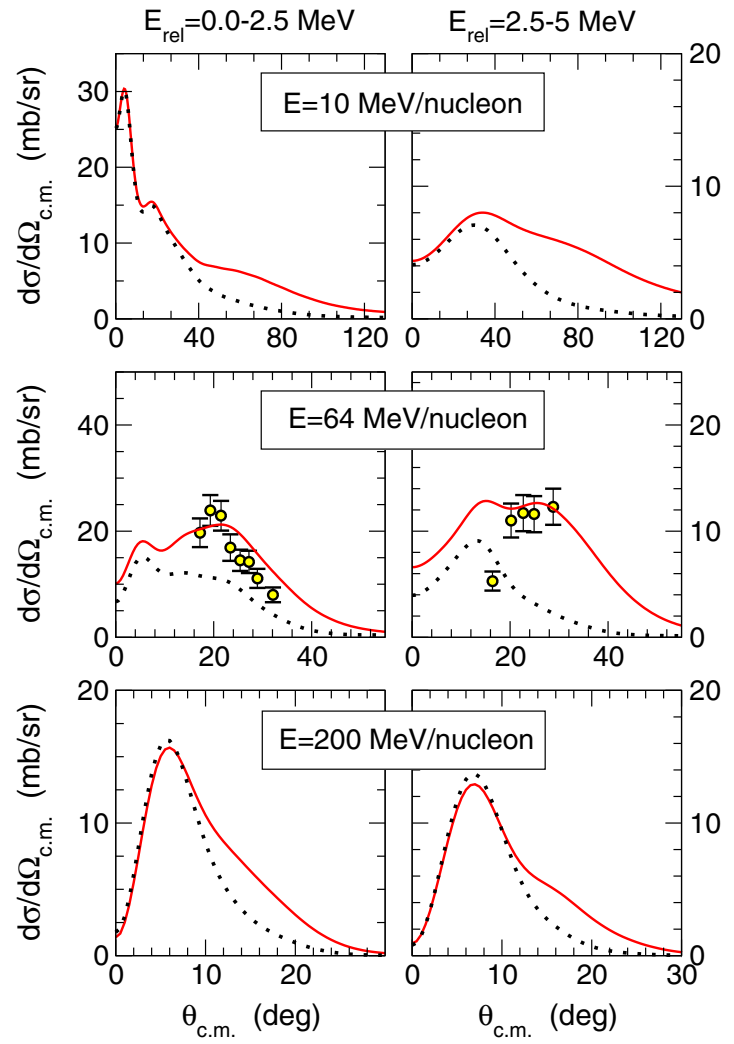

FIG. 3. (Color online) Energy dependence of the core excitation effects in the breakup of ${ }^{11} \mathrm{Be}$ on protons. The top, middle, and bottom rows correspond to the bombarding energies 10, 64, and $200 \mathrm{MeV} /$ nucleon, respectively. The left and right panels are for the neutron-core energy intervals $E_{\text {rel }}=0-2.5 \mathrm{MeV}$ and $E_{\text {rel }}=2.5-$ $5 \mathrm{MeV}$, respectively. In each panel, the solid line is the full XCDCC calculation, whereas the dotted line is the XCDCC calculation without deformation in the $p+{ }^{10} \mathrm{Be}$ potential.

the parameter $\gamma=1.8 \mathrm{fm}^{1 / 2}$ was used, although additional tests were done with other choices to verify the independence and stability of the results with respect to parameters $b$ and $\gamma$. After diagonalization, only eigenstates below $13 \mathrm{MeV}$ were retained for the coupled-channels calculations. We verified that including eigenstates up to $14 \mathrm{MeV}$ had a very small effect on the studied observables.

The calculated differential quasielastic cross section is compared with the data in Fig. 4. The dotted line is the XCDCC calculation neglecting the coupling to the breakup channels, that is, including only the ${ }^{11} \mathrm{Be}$ ground state and first excited state. As expected, this calculation largely fails to describe the data. The solid line is the full XCDCC calculation. This calculation describes well the data in the full angular range. We have also included the result obtained with the standard CDCC calculation from Ref. [54]. Except for some small differences around $\theta_{\text {c.m. }} \approx 30^{\circ}$ it turns out to be very similar.

Although the data of Ref. [52] did not provide the separate contribution of the inelastic cross section for the $1 / 2^{-}$bound state at $E_{x}=320 \mathrm{keV}$, it is worth comparing the values computed with the two methods. In the CDCC calculations of Ref. [54], the total inelastic cross section for the population of this state was about $750 \mathrm{mb}$, whereas in 


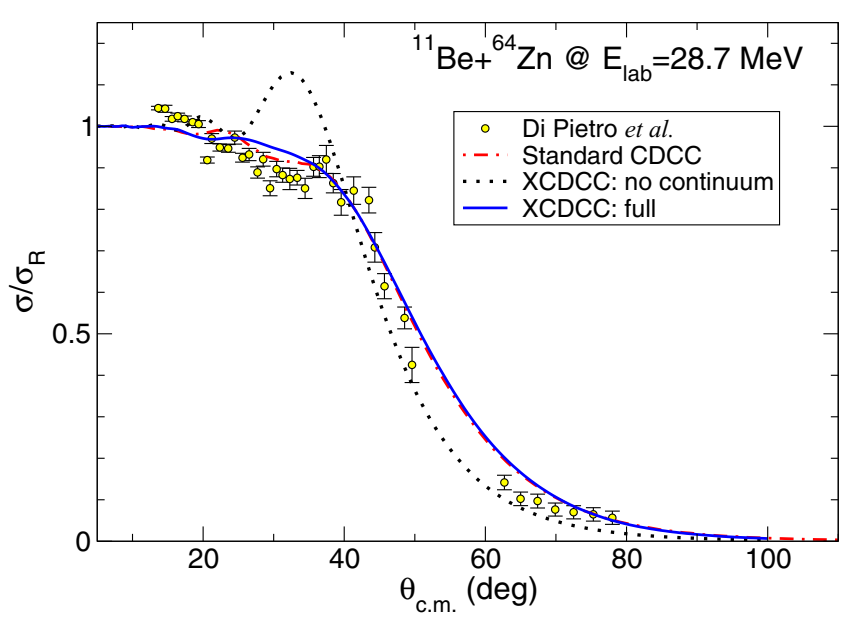

FIG. 4. (Color online) Quasielastic differential cross section, relative to Rutherford, for the scattering of ${ }^{11} \mathrm{Be}$ on ${ }^{64} \mathrm{Zn}$ at $E_{\mathrm{lab}}=$ 28.7 MeV. The dot-dashed line (red online) is the standard CDCC calculation, without core excitation, from Ref. [54]. The solid line (blue online) is the XCDCC calculation. The dotted line is the XCDCC calculation neglecting the coupling to the breakup channels. Experimental data are from Ref. [52].

the XCDCC calculation this value is reduced to $\sim 566 \mathrm{mb}$. The difference can be understood comparing the values of the electric transition probability $B\left(E 1\right.$; g.s. $\left.\rightarrow 1 / 2^{-}\right)$for these two models. The single-particle model used in Ref. [54] yields $B\left(E 1\right.$; g.s. $\left.\rightarrow 1 / 2^{-}\right)=0.260 \mathrm{e}^{2} \mathrm{fm}^{2}$, whereas for the PRM model used here it is $B\left(E 1\right.$; g.s. $\left.\rightarrow 1 / 2^{-}\right)=0.140 e^{2} \mathrm{fm}^{2}$. This value is in better agreement with the experimental one, $B\left(E 1\right.$; g.s. $\left.\rightarrow 1 / 2^{-}\right)=0.116 e^{2} \mathrm{fm}^{2}[56]$, so we expect that the inelastic cross section calculated with XCDCC be more realistic than that obtained with the standard CDCC method.

The same experiment also provided the inclusive ${ }^{10} \mathrm{Be}$ angular distribution. In Fig. 5 we compare the data from Ref. [52]

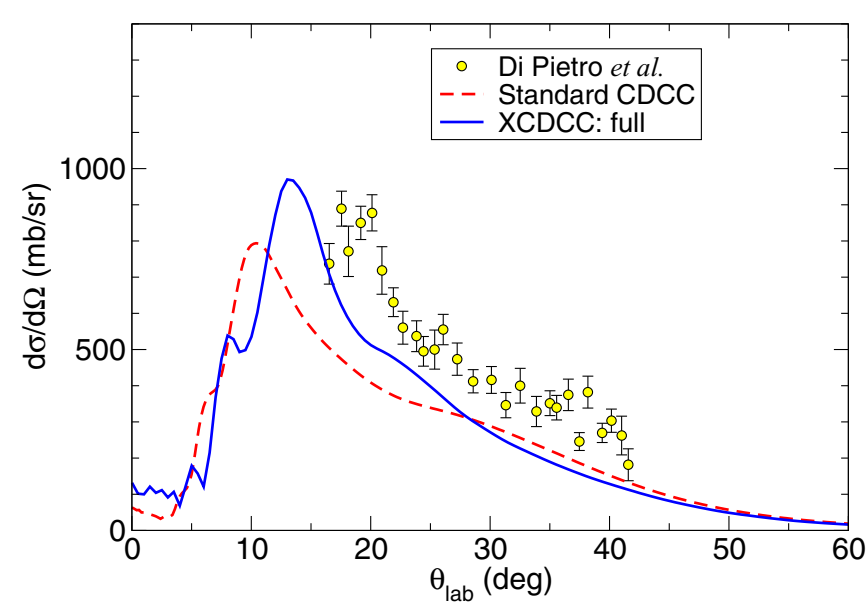

FIG. 5. (Color online) Differential cross section, as a function of the laboratory angle, for the ${ }^{10} \mathrm{Be}$ fragments resulting from the breakup of ${ }^{11} \mathrm{Be}$ on ${ }^{64} \mathrm{Zn}$ at $E_{\text {lab }}=28.7 \mathrm{MeV}$. The dashed line (red online) is the standard CDCC calculation, without core excitation, from Ref. [54]. The solid line (blue online) is the XCDCC calculation. Experimental data are from Ref. [52]. with the present XCDCC calculations and the standard CDCC calculations from Ref. [54]. It is worth noting that the data are referred to the ${ }^{10} \mathrm{Be}$ laboratory angle. The calculation of this observable within the XCDCC framework would require an appropriate kimematical transformation, similar to that developed in Ref. [57] for the standard CDCC method, but this formalism is not yet available for XCDCC. Consequently, we perform an approximate transformation, approximating the ${ }^{10} \mathrm{Be}$ scattering angle by the ${ }^{11} \mathrm{Be}^{*}$ scattering angle.

The XCDCC calculation is found to be larger than the CDCC result. This increase improves the agreement with the data of Ref. [52] although some underestimation is still observed. This remaining discrepancy could be due to the limitations of the ${ }^{11} \mathrm{Be}$ model used in the XCDCC calculations but also to the contribution of nonelastic breakup events in the data. It is worth noting that the CDCC and XCDCC methods provide only the so-called elastic breakup component, that is, the projectile dissociation in which both the neutron and core survive and the target is left in the ground state. However, since the neutrons were not detected in the experiment of Ref. [52], the data might contain also contributions from other processes involving the absorption of the neutron by the target and/or the target excitation.

In the case of ${ }^{11} \mathrm{Be}+p$ breakup at intermediate energies, we found that the deformed part of the core-target interaction gives rise to an increase of the breakup cross sections. Now we study the effect of these terms in the ${ }^{11} \mathrm{Be}+{ }^{64} \mathrm{Zn}$ case. For this purpose, we compare in Fig. 6 the full XCDCC calculation, described above, with another XCDCC calculation, in which the ${ }^{10} \mathrm{Be}+{ }^{64} \mathrm{Zn}$ potential is described with the central optical potential of Refs. [52,54]. Both calculations give almost identical results for the quasielastic and breakup data. This result indicates that, at these low incident energies (a few MeV per nucleon) and for medium-mass targets, the dynamic core excitation effect due to the core-target potential is well represented by an optical potential describing the corresponding elastic data. Consequently, at these energies, the main effect of core excitation comes from the admixtures of core-excited components in the projectile wave functions.

\section{Application to ${ }^{11} \mathrm{Be}+{ }^{208} \mathrm{~Pb}$ breakup}

As a final example, we consider the reaction of ${ }^{11} \mathrm{Be}$ on a ${ }^{208} \mathrm{~Pb}$ target. This reaction has been measured by several groups [58-60] at intermediate energies (several tens of $\mathrm{MeV}$ per nucleon) with the aim of obtaining information on the dipole Coulomb response of ${ }^{11} \mathrm{Be}$ as well as on the amount of $s$-wave component in the ground state. We have performed XCDCC calculations at $69 \mathrm{MeV} / \mathrm{u}$, which corresponds to the energy of the experiment performed at RIKEN by Fukuda et al. [60].

Continuum states with $J_{p}=1 / 2^{ \pm}, 3 / 2^{ \pm}$, and $5 / 2^{+}$were considered (test calculations revealed that the effect of the $5 / 2^{-}$states is negligible for the studied angles). These states were generated with a THO basis with $N=15$ states and $\gamma=1.8 \mathrm{fm}^{1 / 2}(b=1.6 \mathrm{fm}$ as in the preceding cases). After diagonalization, all eigenstates below $8 \mathrm{MeV}$ were retained for the coupled-channels calculation. 

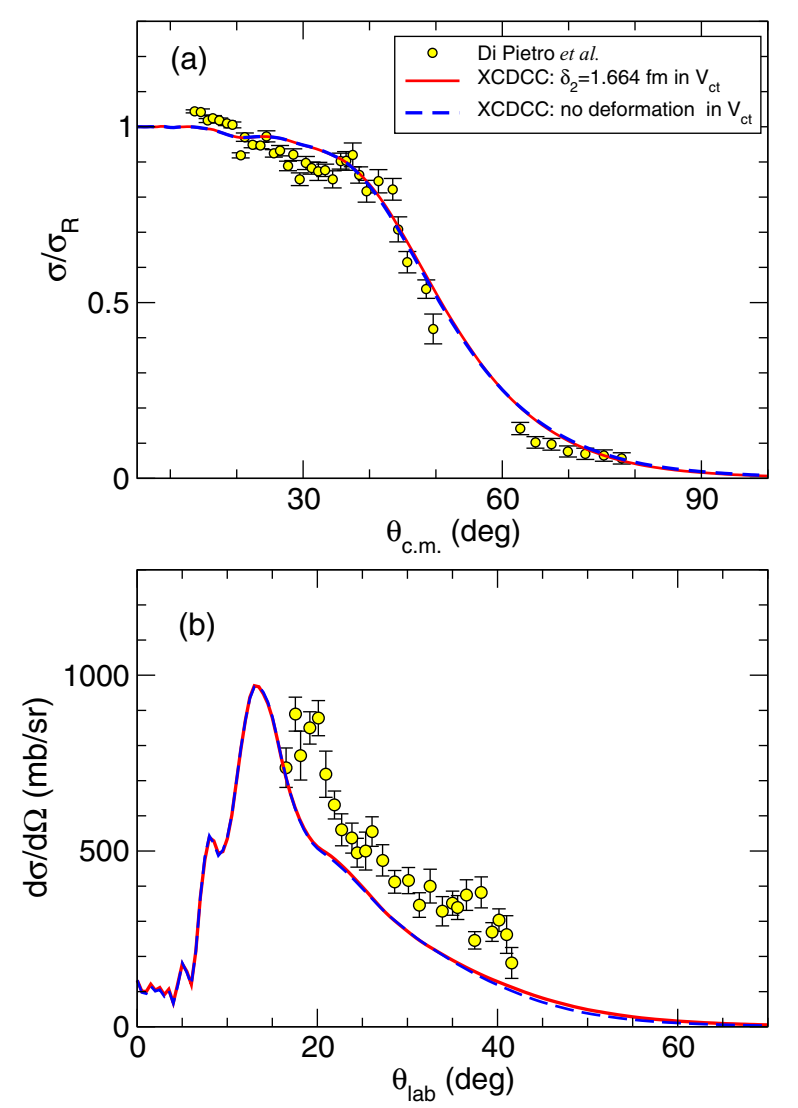

FIG. 6. (Color online) Quasielastic differential cross section (top) and breakup differential cross section (bottom) for ${ }^{11} \mathrm{Be}$ on ${ }^{64} \mathrm{Zn}$ at $E_{\text {lab }}=28.7 \mathrm{MeV}$, compared with XCDCC calculations, for different choices of the ${ }^{10} \mathrm{Be}+{ }^{64} \mathrm{Zn}$ potential. See text for further details.

For the neutron-target interaction we used the parametrization of Koning and Delaroche [61]. The central part of the core-target potential was taken from Ref. [62] (first line of Table III). As in the previous case, this potential is deformed with a deformation length of $\delta_{2}=1.664 \mathrm{fm}$. At these relatively high energies the breakup process is essentially a one-step mechanism connecting the ground state directly with the breakup channels. Moreover, at the very forward angles measured in the experiment of Ref. [60] one expects that the breakup is largely dominated by the dipole Coulomb couplings. Consequently, at these angles the most strongly coupled breakup states will be the $1 / 2^{-}$and $3 / 2^{-}$. These states cannot be populated by the dynamic core excitation mechanism in first order since the quadrupole nature of this excitation connects the ground state with positive-parity continuum states. As a result, for this reaction (and in general for other reactions induced by weakly bound nuclei on heavy targets) the main core excitation effect is due to the presence of core excitation admixtures in the projectile states.

The XCDCC calculations are compared with the data of Ref. [60] in Fig. 7. The solid line is the full calculation. Since the experimental distribution was integrated for relative $n-{ }^{10} \mathrm{Be}$ energies below $5 \mathrm{MeV}$, the theoretical result was obtained adding the angular distributions for positive-energy

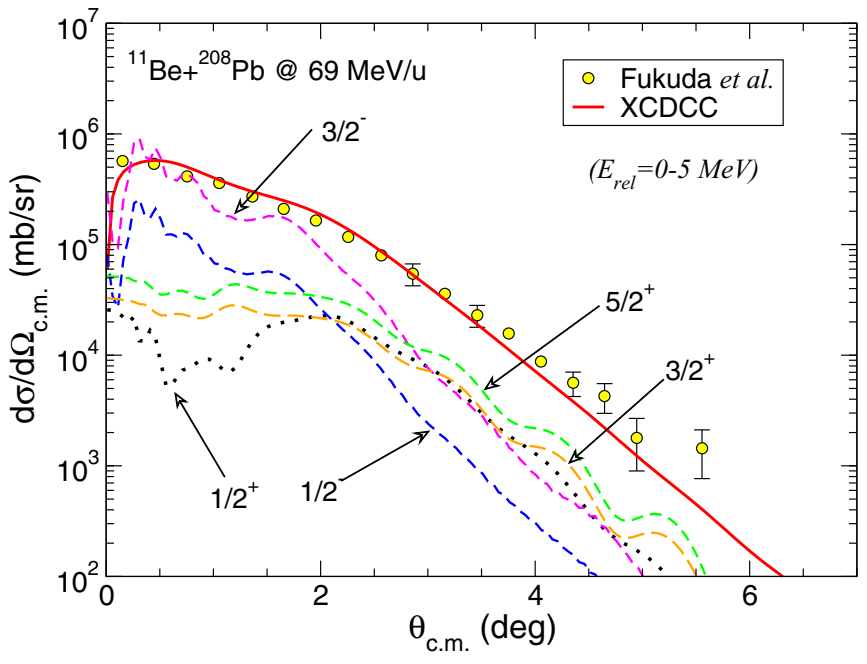

FIG. 7. (Color online) Breakup differential cross section for ${ }^{11} \mathrm{Be}$ on ${ }^{208} \mathrm{~Pb}$ at $E_{\text {lab }}=69 \mathrm{MeV} /$ nucleon, integrated in the $n-{ }^{10} \mathrm{Be}$ relative energy up to $5 \mathrm{MeV}$. The data are from Ref. [60]. The lines are XCDCC calculations described in the text. The full calculation (solid line) has been convoluted with the experimental angular resolution for a meaningful comparison with the data.

pseudostates lying below this energy. The final distribution was convoluted with the experimental angular resolution quoted in Ref. [60]. This calculation is found to be in very good agreement with the data (solid line in Fig. 7). It is worth noting that no scaling factor is introduced in the calculation. The analysis of this kind of experiment is usually done assuming single-particle states for the initial and final states. The final result is then renormalized by a scaling factor which, in the present case, can be interpreted as the spectroscopic factor for the ${ }^{10} \mathrm{Be} \otimes s_{1 / 2}$ configuration in the ground-state wave function. In our calculations, this spectroscopic factor is

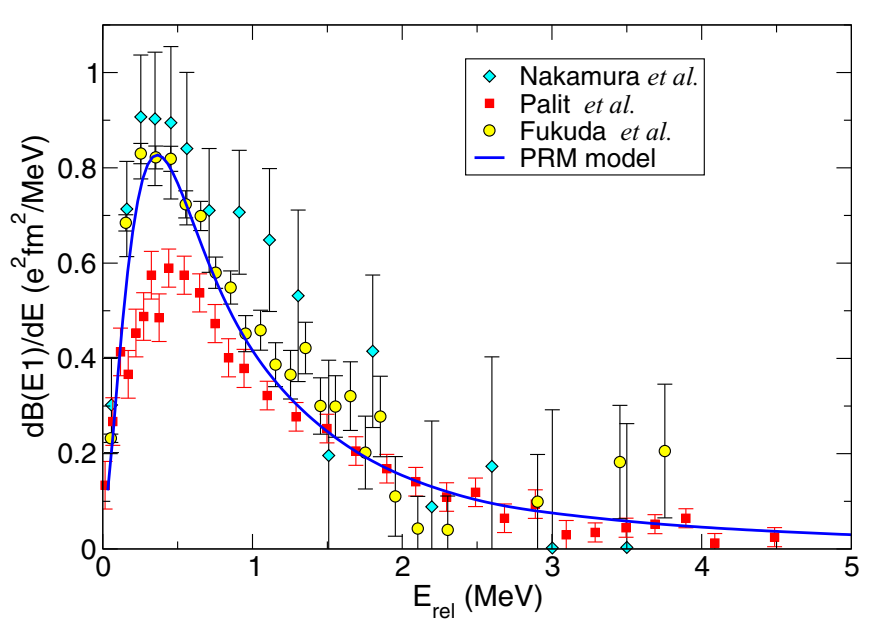

FIG. 8. (Color online) Dipole strength distribution for ${ }^{11} \mathrm{Be}$ deduced from Coulomb breakup experiments (diamonds [58], squares [59], and circles [60]) and from the PRM model of Ref. [36]. The latter has been convoluted with the energy resolution corresponding to the experiment of Ref. [60]. 
already included in the description of the ground-state wave function.

To illustrate the dominance of the dipole excitation mechanism, we have plotted also in Fig. 7 the separate contribution of the $1 / 2^{ \pm}, 3 / 2^{ \pm}$, and $5 / 2^{+}$states. It can be seen that, at sufficiently small angles, the breakup is largely dominated by the coupling to the dipole states and, in particular, to the $3 / 2^{-}$ states.

This dominance of the dipole Coulomb couplings supports the procedure followed in Ref. [60] to extract the $B(E 1)$ response of the ${ }^{11} \mathrm{Be}$ nucleus from the analysis of these exclusive breakup data. In Ref. [60] this was done by comparing the breakup data with first-order semiclassical calculations. The extracted $B(E 1)$ distribution, quoted from Ref. [63], is compared in Fig. 8 with the theoretical $B(E 1)$ distribution obtained with the PRM model adopted in our XCDCC calculations (solid line). We include also the experimental distributions from Refs. [58,59] deduced from similar Coulomb dissociation experiments. It is seen that the theoretical $B(E 1)$ distribution agrees very well with the experimental $B(E 1)$ distribution from Ref. [60], and this explains also the good agreement in the corresponding breakup cross sections.

\section{SUMMARY AND CONCLUSIONS}

To summarize, we have studied the scattering of a twobody halo nucleus (core plus a valence particle) on an inert target within an extended version of the continuum-discretized coupled-channels (XCDCC) formalism. The method takes into account the effect of core excitation in the structure of the projectile, by allowing the inclusion of core-excited components in the projectile states, and also in the dynamics of the reaction, by allowing core excitation and de-excitation during the collision.

The projectile states are described in the weak-coupling limit. Thus, the states of the composite system are expanded as a superposition of products of single-particle configurations and core states. The energies and wave functions of the projectile are calculated using the pseudostate (PS) method, that is, diagonalizing the model Hamiltonian on a basis of square-integrable functions. For the relative motion between the valence particle and the core, we use the analytical transformed harmonic oscillator (THO) basis used in previous works [28].

The method has been applied to the scattering of ${ }^{11} \mathrm{Be}$ on several targets. The ${ }^{11} \mathrm{Be}$ nucleus is described in a simple particle-rotor model, in which the ${ }^{10} \mathrm{Be}$ core is assumed to have a permanent axial deformation with $\beta_{2}=0.67$ [36]. The core-target interaction is obtained by deforming a central phenomenological potential.

To study the dependence of core excitation on the target mass, we have performed calculations for three different targets: ${ }^{1} \mathrm{H},{ }^{64} \mathrm{Zn}$, and ${ }^{208} \mathrm{~Pb}$ at incident energies for which experimental data exist.

In the ${ }^{11} \mathrm{Be}+p$ reaction, the calculations reproduce well the breakup data from Shrivastava et al. [44] corresponding to an incident energy of $64 \mathrm{MeV} /$ nucleon. The XCDCC results are qualitatively similar to those found in previous studies, using a no-recoil XDWBA approximation $[13,14]$. In particular, we confirm the importance of the dynamic core excitation mechanism, due to the noncentral part of the core-target interaction, for the excitation of the low-lying $5 / 2^{+}$and $3 / 2^{+}$ resonances. Moreover, higher order couplings are found to be non-negligible and therefore should be taken into account for an accurate description of similar reactions. In particular, inclusion of breakup beyond first order is found to improve the agreement in the absolute cross section at excitation energies around the $5 / 2_{1}^{+}$resonance.

The ${ }^{11} \mathrm{Be}+{ }^{64} \mathrm{Zn}$ reaction has been studied at $28.7 \mathrm{MeV}$, for which quasielastic and inclusive breakup data are available [52]. The experimental quasielastic cross sections are well reproduced at all angles, except for some slight overestimation at $\theta_{\text {c.m }} \approx 30^{\circ}$. The XCDCC result turns out to be very close to the standard CDCC calculation from Ref. [52]. On the other hand, the inclusive breakup cross sections are larger than those found in the standard CDCC calculations, being in better agreement with the data from Ref. [52]. For this medium-mass target, the dynamic core excitation mechanism is found to be small and the full calculations can be simulated using a central core-target potential fitted to the ${ }^{10} \mathrm{Be}+{ }^{64} \mathrm{Zn}$ elastic data.

Finally, we have presented calculations for the ${ }^{11} \mathrm{Be}+{ }^{208} \mathrm{~Pb}$ reaction at $69 \mathrm{MeV} / \mathrm{u}$. The calculated breakup angular distribution is found to reproduce very well the data from Ref. [60]. For this heavy target, and at very small angles, the breakup is dominated by the dipole Coulomb couplings connecting the ground state with the dipole $\left(1 / 2^{-}\right.$and $\left.3 / 2^{-}\right)$continuum states. In our model, these states cannot be populated by a direct core excitation mechanism, and hence core excitation enters only through the admixture of different core and valence configurations in the projectile wave functions. These admixtures are nevertheless very important to account for the correct normalization of the data.

In summary, the effect of core excitation in the structure is found to be important for all targets. However, the dynamic core excitation mechanism is important for light targets (for which the dipole excitations are small compared to the quadrupole collective excitations of the core) at all incident energies explored here. Although all the calculations presented in this work have been performed for the ${ }^{11} \mathrm{Be}$ nucleus, we believe that the results can be extrapolated to other weakly bound nuclei and, consequently, the effects discussed here should be taken in consideration for an accurate description and interpretation of the data.

\section{ACKNOWLEDGMENTS}

We are grateful to I. J. Thompson, N. C. Summers, and F. M. Nunes for very useful discussions and feedback regarding the XCDCC formalism and for letting us using an unpublished version of FRESCO for comparison. This work has been partially supported by the Spanish Ministerio de Economía y Competitividad and FEDER funds under Projects No. FIS2011-28738-c02-01, No. FPA2009-07653, and No. FPA2009-08848; by the Spanish Consolider-Ingenio 2010 Programme CPAN (CSD2007-00042); and by Junta de Andalucía (FQM160, P11-FQM-7632). One of us (R.D.) acknowledges support by the Fundação para a Ciência e a Tecnologia (FCT) Grant No. SFRH/BPD/78606/2011. 
[1] G. H. Rawitscher, Phys. Rev. C 9, 2210 (1974).

[2] N. Austern, Y. Iseri, M. Kamimura, M. Kawai, G. Rawitscher, and M. Yahiro, Phys. Rep. 154, 125 (1987).

[3] P. Banerjee and R. Shyam, Phys. Rev. C 61, 047301 (2000).

[4] J. A. Tostevin, S. Rugmai, and R. C. Johnson, Phys. Rev. C 57, 3225 (1998).

[5] L. D. Faddeev, Zh. Eksp. Theor. Fiz. 39, 1459 (1960) [Sov. Phys. JETP 12, 1014 (1961)].

[6] E. O. Alt, P. Grassberger, and W. Sandhas, Nucl. Phys. B 2, 167 (1967).

[7] S. Typel and G. Baur, Phys. Rev. C 50, 2104 (1994).

[8] H. Esbensen and G. F. Bertsch, Nucl. Phys. A 600, 37 (1996).

[9] T. Kido, K. Yabana, and Y. Suzuki, Phys. Rev. C 50, R1276 (1994).

[10] S. Typel and G. Baur, Phys. Rev. C 64, 024601 (2001).

[11] P. Capel, G. Goldstein, and D. Baye, Phys. Rev. C 70, 064605 (2004).

[12] A. García-Camacho, A. Bonaccorso, and D. M. Brink, Nucl. Phys. A 776, 118 (2006).

[13] R. Crespo, A. Deltuva, and A. M. Moro, Phys. Rev. C 83, 044622 (2011).

[14] A. M. Moro and R. Crespo, Phys. Rev. C 85, 054613 (2012).

[15] A. M. Moro and J. A. Lay, Phys. Rev. Lett. 109, 232502 (2012).

[16] K. Horii, M. Takashina, T. Furumoto, Y. Sakuragi, and H. Toki, Phys. Rev. C 81, 061602 (2010).

[17] R. C. Johnson, J. S. Al-Khalili, and J. A. Tostevin, Phys. Rev. Lett. 79, 2771 (1997).

[18] N. C. Summers, F. M. Nunes, and I. J. Thompson, Phys. Rev. C 74, 014606 (2006).

[19] N. C. Summers, F. M. Nunes, and I. J. Thompson, Phys. Rev. C 89, 069901(E) (2014).

[20] N. C. Summers, F. M. Nunes, and I. J. Thompson (private communication).

[21] T. Matsumoto, T. Kamizato, K. Ogata, Y. Iseri, E. Hiyama, M. Kamimura, and M. Yahiro, Phys. Rev. C 68, 064607 (2003).

[22] F. Pérez-Bernal, I. Martel, J. M. Arias, and J. Gómez-Camacho, Few-Body Syst. Suppl. 13, 217 (2002).

[23] A. M. Moro, J. M. Arias, J. Gómez-Camacho, and F. PérezBernal, Phys. Rev. C 80, 054605 (2009).

[24] T. Matsumoto, E. Hiyama, M. Yahiro, K. Ogata, Y. Iseri, and M. Kamimura, Nucl. Phys. A 738, 471 (2004).

[25] T. Matsumoto, E. Hiyama, K. Ogata, Y. Iseri, M. Kamimura, S. Chiba, and M. Yahiro, Phys. Rev. C 70, 061601(R) (2004).

[26] T. Matsumoto, T. Egami, K. Ogata, Y. Iseri, M. Kamimura, and M. Yahiro, Phys. Rev. C 73, 051602(R) (2006).

[27] M. Rodríguez-Gallardo, J. M. Arias, J. Gómez-Camacho, R. C. Johnson, A. M. Moro, I. J. Thompson, and J. A. Tostevin, Phys. Rev. C 77, 064609 (2008).

[28] J. A. Lay, A. M. Moro, J. M. Arias, and J. Gómez-Camacho, Phys. Rev. C 85, 054618 (2012).

[29] S. Karataglidis, K. Amos, and B. G. Giraud, Phys. Rev. C 71, 064601 (2005).

[30] A. Bohr and B. Mottelson, Nuclear Structure (W. A. Benjamin, New York, 1969).

[31] T. Tamura, Rev. Mod. Phys. 37, 679 (1965).

[32] I. J. Thompson and F. M. Nunes, Nuclear Reactions for Astrophysics (Cambridge University Press, Cambridge, 2009).

[33] S. Fortier et al., Phys. Lett. B 461, 22 (1999).
[34] J. S. Winfield et al., Nucl. Phys. A 683, 48 (2001).

[35] F. Cappuzzello, A. Cunsolo, S. Fortier, A. Foti, M. Khaled, H. Laurent, H. Lenske, J. M. Maison, A. L. Melita, C. Nociforo et al., Phys. Lett. B 516, 21 (2001).

[36] F. M. Nunes, J. A. Christley, I. J. Thompson, R. C. Johnson, and V. D. Efros, Nucl. Phys. A 609, 43 (1996).

[37] T. Tarutina and M. S. Hussein, Phys. Rev. C 70, 034603 (2004).

[38] T. Tarutina, A. R. Samana, F. Krmpotic, and M. S. Hussein, Braz. J. Phys. 36, 1349 (2006).

[39] S. Karataglidis, K. Amos, P. Fraser, L. Canton, and J. Svenne, Nucl. Phys. A 813, 235 (2008).

[40] Y. Kanada-En'yo and H. Horiuchi, Phys. Rev. C 66, 024305 (2002).

[41] J. A. Lay, A. M. Moro, J. M. Arias, and Y. Kanada-En'yo, Phys. Rev. C 89, 014333 (2014).

[42] C. Forssén, P. Navrátil, W. E. Ormand, and E. Caurier, Phys. Rev. C 71, 044312 (2005).

[43] P. Descouvemont and M. S. Hussein, Phys. Rev. Lett. 111, 082701 (2013).

[44] A. Shrivastava et al., Phys. Lett. B 596, 54 (2004).

[45] J. H. Kelley et al., Nucl. Phys. A 880, 88 (2012).

[46] R. L. Varner, W. J. Thompson, T. L. McAbbe, E. J. Ludwig, and T. B. Clegg, Phys. Rep. 201, 57 (1991).

[47] H. Iwasaki, T. Motobayashi, H. Akiyoshi, Y. Ando, N. Fukuda, H. Fujiwara, Z. Fulop, K. I. Hahn, Y. Higurashi, M. Hirai et al., Phys. Lett. B 481, 7 (2000).

[48] S. Raman, C. H. Malarkey, W. T. Milner, C. W. Nestor, Jr., and P. H. Stelson, At. Data Nucl. Data Tables 36, 1 (1987).

[49] N. C. Summers and F. M. Nunes, Phys. Rev. C 76, 014611 (2007).

[50] J.-P. Jeukenne, A. Lejeune, and C. Mahaux, Phys. Rev. C 16, 80 (1977).

[51] M. Takashina and Y. Kanada-Enyo, Phys. Rev. C 77, 014604 (2008).

[52] A. Di Pietro, G. Randisi, V. Scuderi, L. Acosta, F. Amorini, M. J. G. Borge, P. Figuera, M. Fisichella, L. M. Fraile, J. GomezCamacho et al., Phys. Rev. Lett. 105, 022701 (2010).

[53] N. Keeley, N. Alamanos, K. W. Kemper, and K. Rusek, Phys. Rev. C 82, 034606 (2010).

[54] A. Di Pietro, V. Scuderi, A. M. Moro, L. Acosta, F. Amorini, M. J. G. Borge, P. Figuera, M. Fisichella, L. M. Fraile, J. GomezCamacho et al., Phys. Rev. C 85, 054607 (2012).

[55] T. Druet and P. Descouvemont, Eur. Phys. J. A 48, 147 (2012).

[56] D. J. Millener, J. W. Olness, E. K. Warburton, and S. S. Hanna, Phys. Rev. C 28, 497 (1983).

[57] J. A. Tostevin, F. M. Nunes, and I. J. Thompson, Phys. Rev. C 63, 024617 (2001).

[58] T. Nakamura, S. Shimoura, T. Kobayashi, T. Teranishi, K. Abe, N. Aoi, Y. Doki, M. Fujimaki, N. Inabe, N. Iwasa et al., Phys. Lett. B 331, 296 (1994).

[59] R. Palit et al., Phys. Rev. C 68, 034318 (2003).

[60] N. Fukuda et al., Phys. Rev. C 70, 054606 (2004).

[61] A. Koning and J. Delaroche, Nucl. Phys. A 713, 231 (2003).

[62] P. Capel, D. Baye, and V. S. Melezhik, Phys. Rev. C 68, 014612 (2003).

[63] T. Nakamura and Y. Kondo, in Clusters in Nuclei, edited by C. Beck, Lecture Notes in Physics 848 (Springer-Verlag, Berlin, 2012), Vol. 2. 